IS THERE EVIDENCE OF ANISOTROPY IN THE EXPANSION OF THE UNIVERSE? Vera C. Rubin

Department of Terrestrial Magnetism,Carnegie Institution of Washington

Un échantillon idéal pour la recherche d'une éventuelle anisotropie dans l'expansion de Hubble doit couvrir la totalité de la surface du ciel, de plus les magnitudes et les vitesses ne doivent pas être entachées d'erreurs dépendant de la position des objets choisis. Très peu d'échantillons satisfont ces critères. Toutefois les échantillons de galaxies Sc I, de galaxies $E$ et $S O$, et d'amas riches mettent en évidence une anisotropie provenant soit d'une vitesse du groupe local $\sim 500 \mathrm{kms}^{-1},\left(\mathrm{~V}_{\odot} \sim 600 \mathrm{kms}^{-1}\right)$, soit du fait que $\mathrm{H}$ varie de $20 \%$ d'un point à l'autre du ciel, soit du fait que $m$ ou $M_{o}$ varie de $0.4 \mathrm{~m}$. Des explications moins conventionnelles ne semblent pas nécessaires. Seul le mouvement du groupe local est compatible avec les données de l'échantillon de SC I les plus proches. Compte-tenu du mouvement de l'observateur, l'expansion de Hubble ne présente pas d'anisotropie supérieure à $5 \%$. Toutefois, cette interprétation n'est pas compatible avec le rayonnement à $3^{\circ} \mathrm{K}$ qui impose $V_{\odot}<300 \mathrm{kms}^{-1}$.

\title{
I. INTRODUCTION
}

In the 50 years since Hubble (1929) published the first redshiftmagnitude diagram, observational cosmology has been concerned with the evaluation of two numbers, $H$ and $q_{O}$. Only in the past few years have there been observational attempts to answer the less orthodox question, "Is the Hubble expansion isotropic as observed from the sun?" Observational data are not sufficiently precise to detect anisotropies of a few percent, but anisotropies of order $50 \%$ would surely have been noted in prior studies of magnitudes and velocities. Modern observations could detect anisotropies of perhaps $10 \%$ in $\mathrm{H}$ across the sky. It is an anisotropy of this order across the sky which we here discuss.

Following the initial work of Rubin, Ford, and Rubin (1973), which suggested a non-isotropic distribution in the velocities and magnitudes of faint SC I galaxies, there has been a rash of papers detecting, supporting, questioning, dismissing, and refuting such an effect for a variety of extragalactic objects. However, few studies fulfill what I would set forth as the minimum requirements of any observational sample for an examination of possible anisotropies:

1) all-sky coverage, 
2) limited velocity (distance) range or ranges,

3) no Malmquist bias (or bias not position dependent),

4) careful evaluation of magnitude corrections, to eliminate regional effects due to extinction in our Galaxy and in the individual galaxies, effects due to varying luminosity classes, effects due to cluster richness, etc.

In the present paper, I shall show how these points relate to a few recent studies, and assess the present observational status for a possible anisotropy. The formulation is made in the following framework. For an allsky sample of a restricted type of galaxy (or other standard candle) with mean absolute magnitude $M_{O}$ and dispersion $\sigma_{O_{0}}$, the relation between apparent magnitude $m$, galactocentric velocity $V$, the Hubble constant $H$ and $M_{O}$ is:

$$
\mathrm{HM}=\log \mathrm{V}-0.2 \mathrm{~m}=\log \mathrm{H}-0.2 \mathrm{M}_{\mathrm{O}}-5 \text {. }
$$

We call HM the Hubble modulus; it is a parameter which is proportional to $\log \mathrm{H}$ or to $\mathrm{M}_{\mathrm{O}^{\prime}}$ and whose variation across the sky will map the regional variation of $\log \mathrm{H}$ or $\mathrm{M}_{\mathrm{O}}$, if $\mathrm{V}$ and $\mathrm{m}$ have no position dependent errors.

\section{AN ANISOTROPY IN HM FOR SAMPLES AT DIFFERENT DISTANCES}

For an all-sky sample of galaxies for which HM region I $\neq$ HM region II' regions I and II each a significant fraction of the sky separated by $\sim 180^{\circ}$, we can attribute the non-equality of HM to one of the following: a) a motion $\mathrm{V}_{\mathrm{GM}}$ of our Galaxy and the Local Group; b) systematic errors $\Delta \mathrm{m}$ in the magnitude system, or errors in corrections for internal or external extinction; c) a variation $\Delta \mathrm{H}$ in $\mathrm{H}$ (i.e., a true anisotropy in $\mathrm{H}$ ) with angular position on the sky; or d) systematic differences $\Delta M_{0}$ in the intrinsic luminosities of the galaxies. For each of these situations considered individually, Eq. (1) becomes:

$$
\begin{aligned}
& (\mathrm{HM}+\Delta \mathrm{HM})=\log \left(\mathrm{V}+\mathrm{V}_{\mathrm{GM}}\right)-0.2 \mathrm{~m}_{\mathrm{O}}=\log \mathrm{H}-0.2 \mathrm{M}_{\mathrm{O}}-5, \\
& (\mathrm{HM}+\Delta \mathrm{HM})=\log \mathrm{V}-0.2(\mathrm{~m}+\Delta \mathrm{m})=\log \mathrm{H}-0.2 \mathrm{M}_{\mathrm{O}}-5, \\
& (\mathrm{HM}+\Delta \mathrm{HM})=\log \mathrm{V}-0.2 \mathrm{~m}_{\mathrm{O}}=\log (\mathrm{H}+\Delta \mathrm{H})-0.2 \mathrm{M}_{\mathrm{O}}-5, \\
& (\mathrm{HM}+\Delta \mathrm{HM})=\log \mathrm{V}-0.2 \mathrm{~m}_{\mathrm{O}}=\log \mathrm{H}-0.2\left(\mathrm{M}_{\mathrm{O}}+\Delta \mathrm{M}_{\mathrm{O}}\right)-5 .
\end{aligned}
$$

Note that for a simple model (i.e., only one effect considered at a time, magnitude errors and $\Delta H / H$ not distance dependent), only Eq (la) is distance dependent; the Local Group velocity $V_{G M}$ is a smaller fraction of $\log \left(V+V_{G M}\right)$ for increasingly distant galaxies. In contrast, magnitude errors or a variation in $\mathrm{H}$ across the sky produce values of $\mathrm{HM}$ which are distance independent. This is illustrated in Table I. Values of $\mathrm{HM}$ I and HM II are calculated for samples at velocities of 2000 to $25000 \mathrm{~km} \mathrm{~s}^{-1}$. Galaxies 
TABLE I. Predicted values of $\mathrm{HM}=\log \mathrm{V}-0.2 \mathrm{~m}=\log \mathrm{H}-0.2 \mathrm{M}_{\circ}-5 *$

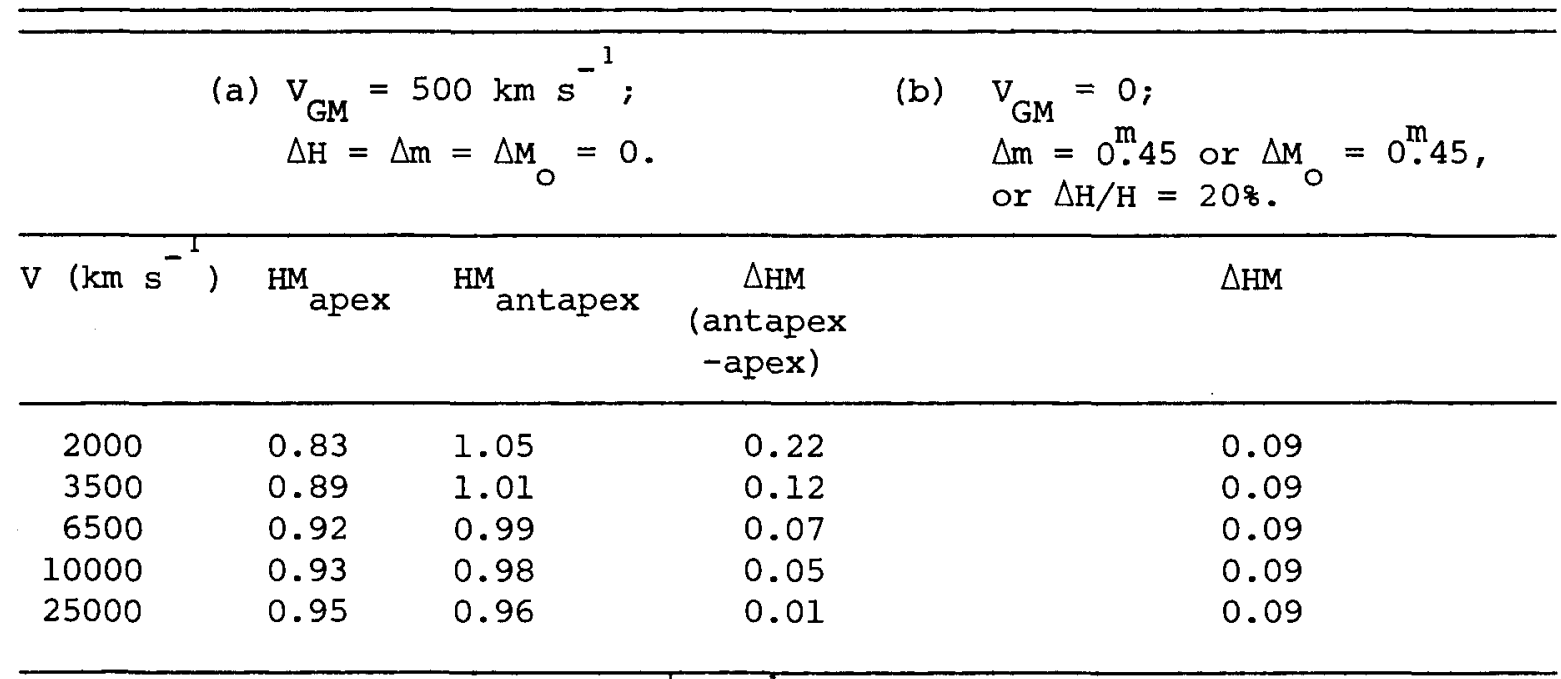

* In calculation, $\mathrm{H}=50 \mathrm{~km} \mathrm{~s}{ }^{-1} \mathrm{Mpc}^{-1} ; \mathrm{M}_{\mathrm{O}}=-21.24$.

with Hubble velocities greater than about $10000 \mathrm{~km} \mathrm{~s}^{-1}$ carry little information concerning a Local Group velocity of $\mathrm{v}_{\mathrm{GM}}{ }^{2500} \mathrm{~km} \mathrm{~s}^{-1}$. Because of this distance dependence, it would be virtually impossible to detect a

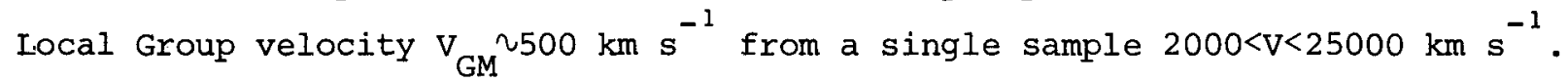
I think it is fair to conclude that studies which include galaxies with a large velocity range and find no significant positional difference in $\mathrm{HM}$ have not made a valid analysis to detect a galactic motion. Conversely, studies which detect a difference in HM of the same amplitude for samples at different distances have detected either a distance independent systematic apparent magnitude error or a variation of $\mathrm{M}_{\mathrm{O}}$ or $\mathrm{H}$ across the sky, but have ruled out a motion of the observer as a cause of the anisotropy.

\section{ELIMINATION OF A MALMQUIST BIAS}

Virtually all galaxy samples are magnitude limited samples, hence suffer from a Malmquist bias. Galaxies at the faint magnitude limit come preferentially from the bright end of the luminosity distribution; the bias results from assigning to each galaxy the mean absolute magnitude $M_{{ }^{\prime}}$, rather than a brighter value. This is illustrated schematically in Fig. 1; galaxies above the Hubble line are intrinsically bright (high HM), and galaxies below the Hubble line are intrinsically faint (low HM).

A biased sample will produce apparent values of $\mathrm{H}$ which increase with distance. Surprisingly, this was elucidated only recently by Teerikorpi $(1975 a, b)$ This understanding should put to rest parabolic redshift laws (de Vaucouleurs 1972, Segal 1975). However, the presence of a bias does not render a sample invalid for a statistical analysis. If the bias is similar 


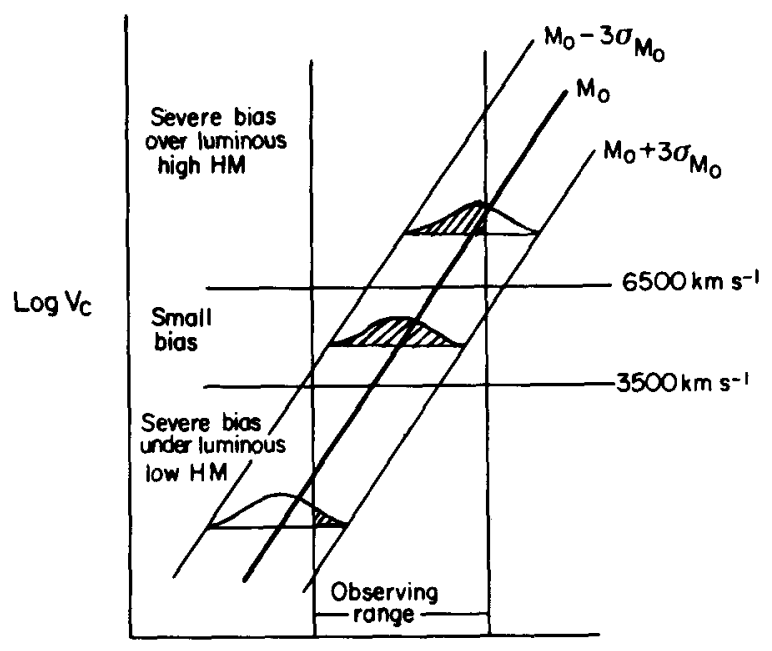

$\mathrm{mc}$
Fig. 1. Schematic Hubble diagram showing effect of limited magnitude observing range. Mean Hubble line (M) and low luminosity $(\mathrm{M}+3 \sigma \mathrm{M})$ ) and high luminosity $\left(\mathrm{M}^{\circ}-3 \sigma \mathrm{M}^{\circ}\right)$ limits are indicated. At low velocity, galaxies of low luminosity are preferentially observed; at high velocity, galaxies of high luminosity are preferentially observed.

on all regions of the sky, then it will not affect the regional variation of HM. If, as is equally likely, the bias has a positional dependence, the bias must be identified and eliminated from the sample. Various procedures for so doing are straightforward. A limited velocity range with minimum bias can be identified. Or the increase in HM with $\mathrm{V}$ (i.e., the bias) can be evaluated, and applied as a correction to $M_{0}$ as a function of V. For spiral galaxies, the high correlation of galaxy luminosity with diameter makes it possible to identify overluminous and underluminous galaxies by their diameters. Values of absolute magnitudes assigned as a function of linear diameters are a significantly closer approximation to true luminosities than is a single mean for the entire sample.

Until a fair assessment of the effect of a Malmquist bias on a sample has been made, it is premature to embark on a study of anisotropies contained in that sample. We show this in more detail below.

\section{CONVENTIONAL CORRECTIONS TO THE APPARENT MAGNITUDES}

To map the variation in HM across the sky for any sample, the apparent magnitudes must be free of position dependent systematic errors, must be corrected for extinction in our Galaxy, internal extinction in each galaxy, variation in absolute magnitude over the range of galaxy classes included, and (for elliptical galaxies in clusters) for the variation in absolute magnitude of the brightest galaxies with cluster richness. Unfortunately, each of these corrections is imperfectly known, and the possibility is very real that an observed variation in $\mathrm{HM}$ is not mapping a large scale cosmological phenomenon, but merely errors in the adopted corrections to the magnitudes. For a large homogeneous sample, these corrections can probably best be obtained 
internally at present. Cosmic values of the corrections are not required, but rather values which are applicable to the sample. For example, if (by some mystical procedure) galaxies in extinction free regions were chosen all over the sky, then the application of conventional extinction corrections would be inappropriate for that study.

If analysis indicates that the value of HM (or equivalently, $M_{0}$ or $\mathrm{H}$ ) varies with luminosity class, with galaxy inclination, or with galactic latitude, it is likely that the adopted corrections are incorrect. These undesirable correlations must be eliminated before analysis for possible anisotropies.

These points have been stressed at some length, because they form important criteria in assessing studies of anisotropies in galaxy magnitudes, velocities, and diameters.

\section{WHAT IS THE EVIDENCE FOR AN ANISOTROPY?}

(a) A survey of Sc I Galaxies

I will discuss our recent results, then go to a selected sample from the 25 or 30 current papers which examine extragalactic samples for anisotropies. In each case, I will attempt to indicate the pitfalls which the author has or has not avoided:

From an all-sky sample of 184 Sc I - Sc II galaxies (Rubin et. al. 1976a, 1976b), the minimum bias subset of $96,3500<\mathrm{V}<6500 \mathrm{~km} \mathrm{~s}^{-1}$, exhibits an anisotropy in HM across the sky of about 10\%, at the 3.60 level. Values of 〈HM〉 increase fairly smoothly from a minimum 〈HM〉 apex $=0.912 \pm 0.012$ at early declination, high right ascension, to a maximum $\left\langle\mathrm{HM}_{\mathrm{a}}\right\rangle$ antapex $=0.995 \pm 0.012,180^{\circ}$ away. Galaxies at early $\alpha$, high $\delta$ have magnitudes which are too faint for their velocities. This is illustrated in Fig. 2, where values of HM, and of $\mathrm{H}$ are plotted as a function of cosine apex distance. These plots are different representations of the same data.

The observed anisotropy implies one of the following (Eq. 1):

a) The Galaxy and the Local Group have a velocity $V_{G M}=454 \pm 125 \mathrm{~km} \mathrm{~s}^{-1}$ toward $l=163^{\circ} \pm 15^{\circ}, b=-11^{\circ} \pm 14^{\circ}$. The galaxy is moving edge on; the leading edge is toward the anticenter of the galaxy;

b) the apparent magnitudes of galaxies in the apex (the hemisphere containing the anticenter) are too faint by $0.4 \pm 0.1$, because of systematic magnitude errors; or, if due to extinction, galaxies toward the anticenter undergo 0.4 more extinction;

c) Galaxies in the apex are intrinsically $0.4 \pm 0.1$ less luminous than 


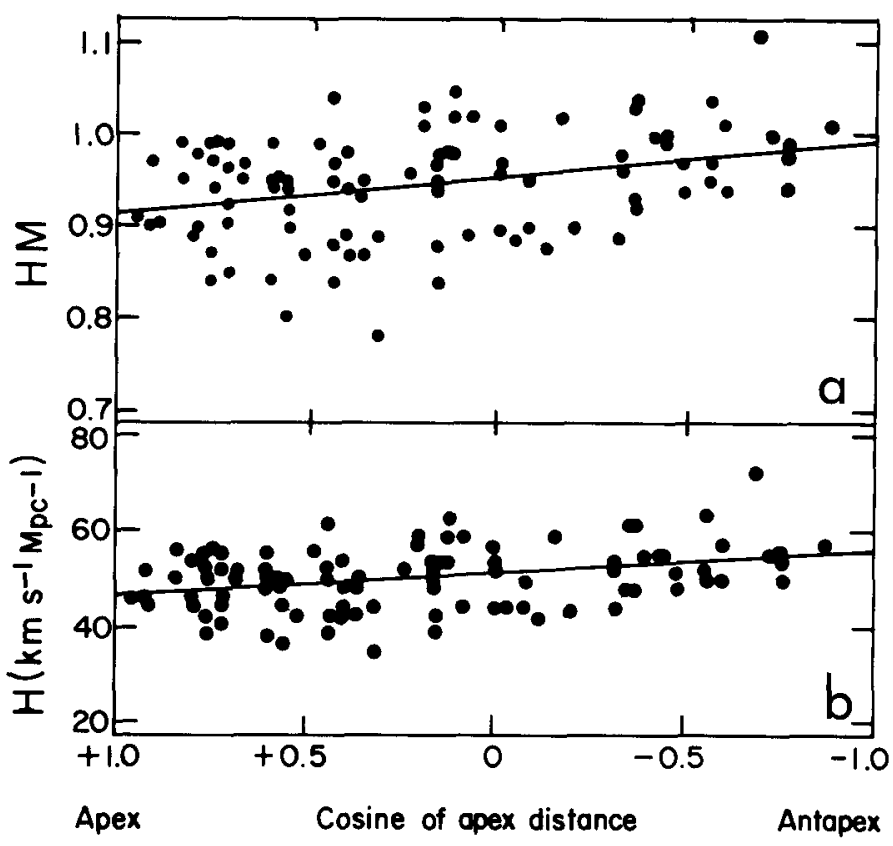

Fig. 2. (a) Variation of $\mathrm{HM}$ as a function of cosine of apex distance; linear least squares $\mathrm{fit}$ is $\mathrm{HM}=$ $0.954 \pm 0.006+(0.042 \pm 0.011) \cos \theta$. (b) Apparent variation of $\mathrm{H}$ as a function of cosine of apex distance; $\mathrm{H}=51.3 \pm 0.7$ $-(4.9 \pm 1.3) \cos \theta$. Effect of motion of observer is to appearently decrease $\mathrm{H}$ to 46 toward apex, and increase $\mathrm{H}$ to 56 toward antapex. This is not a determination of $\mathrm{H}$, for $\mathrm{M}$ is_chosen, so that $\mathrm{H} \sim 50 \mathrm{~km} \mathrm{~s}^{-1} \mathrm{Mpc}^{-1}$.

apex galaxies;

d) $\mathrm{H}$ varies by $20 \%$ from apex to antapex.

The nearer group of 22 galaxies, $1600<\mathrm{V}<3500 \mathrm{~km} \mathrm{~s}^{-1}$ also exhibits a variation in HM across the sky, of the same phase, but of greater amplitude (Fig. 3); 〈HM $\rangle_{\text {apex }}=0.762 \pm 0.027 ;\langle H M\rangle_{\text {antapex }}=0.923 \pm 0.27$. (Note that these values of HM are each lower than the corresponding values in the 3500-6500 $\mathrm{km} \mathrm{s}{ }^{-1}$ sample. The nearby galaxies are underluminous, hence have small HM,

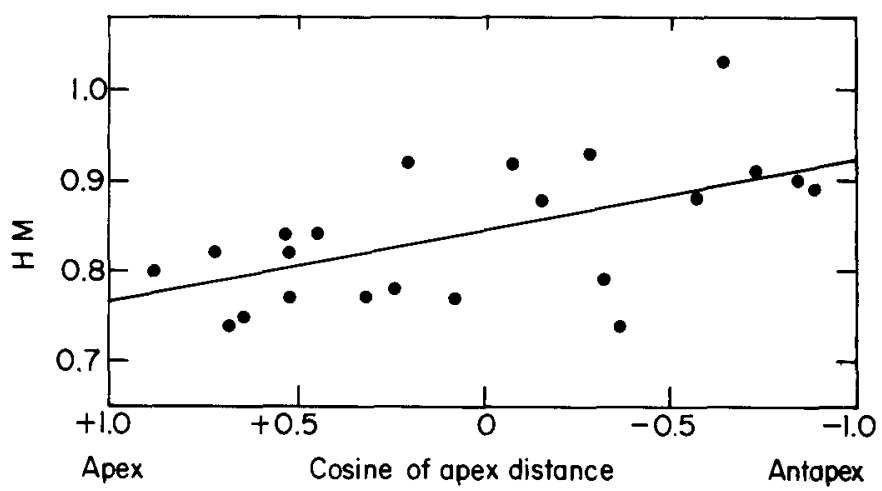

Fig. 3. Variation of $\mathrm{HM}$ as a function of cosine of apex distance for Sc I galaxies $\mathrm{V}<3500 \mathrm{~km} \mathrm{~s}^{-1} ; \mathrm{HM}=$ $0.843 \pm 0.013-(0.080 \pm 0.025) \cos \theta$. Note increased steepness in slope of HM for this nearer sample, compared with Fig. 2a.

but this is of no consequence as the bias has no positional dependence.) The results from this nearer sample imply:

a) $\mathrm{V}_{\mathrm{GM}}=477 \pm 164 \mathrm{~km} \mathrm{~s}^{-1}$ toward $\ell=167^{\circ} \pm 20^{\circ}, \mathrm{b}=+5^{\circ} \pm 20^{\circ}$;

b) $\Delta \mathrm{m}=0.8 \pm 0.2$; or c) $\Delta \mathrm{M}_{\mathrm{O}}=0.8 \pm 0.2$; or d) $\Delta \mathrm{H} / \mathrm{H}=408$.

Only a motion of our galaxy and the Local Group is consistent with the results obtained from ScI galaxies in the two distance groups.

For the Rubin sample, several procedures have been utilized to minimize the bias:

a) Only a restricted velocity subset of galaxies has been studied, the 
subset identified by the variation of $\mathrm{H}$ with $\log \mathrm{V}$ as a minimum bias subset.

b) Under and overluminous galaxies have been identified by their diameters, and $M_{O}$ assigned accordingly. The relation between $M_{0}$ and diameter for the Sc I galaxies (in the minimum bias subset) is shown in Fig. 4.

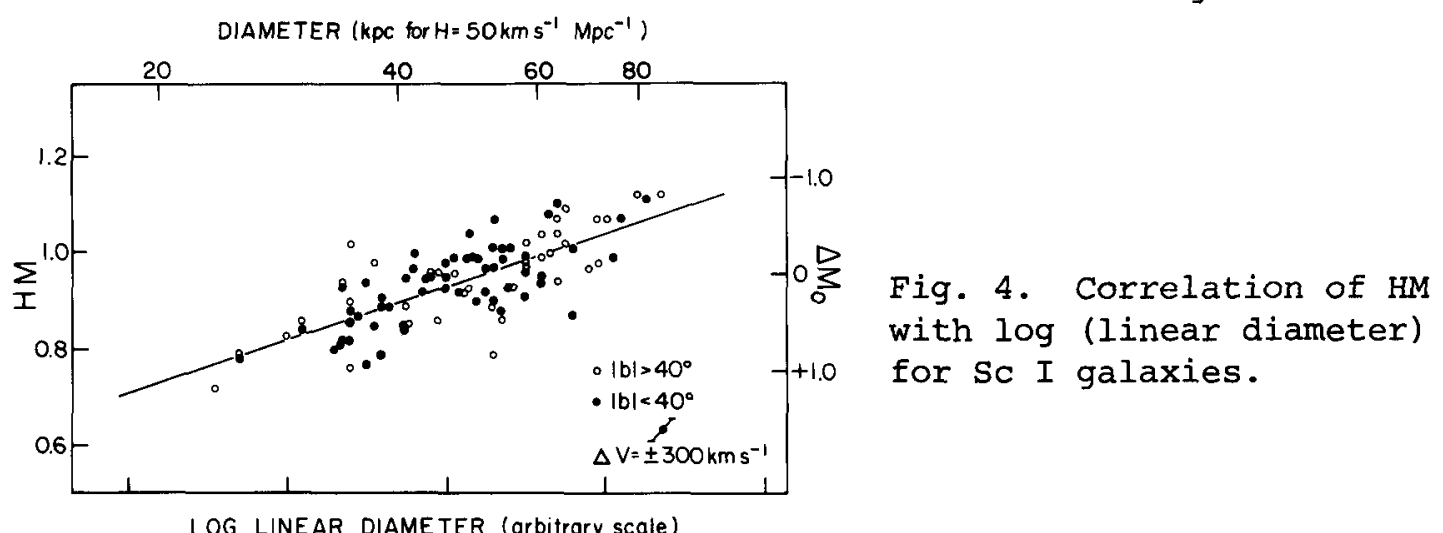

LOG LINEAR DIAMETER (arbitrary scale)

c) A value of $M_{o^{\prime}}$, where $M_{0}=f(\log V)$, has been used for each galaxy, and the analysis repeated. The results are unchanged, at the lo level.

Moreover, the results have been examined to see if the physical parameters differ for apex and antapex galaxies:

a) If the observed anisotropy arises because galaxies in the antapex are 0.4 brighter, they will be 1.5 times as luminous, and $\sim 18 \mathrm{kpc}$ larger (Fig. 4). No such difference is observed. Galaxies in the apex and antapex have the same linear diameters at the $1 \sigma$ level; $1 \sigma=4 \mathrm{kpc}$.

b) On the reasonable assumption that the form of the rotation curve and $M / L$ are similar for all galaxies in our fairly homogeneous sample, then the peak velocity $v_{\max }$ of the rotation curve goes as $L^{1 / 2}$. If antapex galaxies are 1.5 times as luminous, peak rotation curves should be higher by $\sim 40 \mathrm{~km} \mathrm{~s}^{-1}$. We can deduce $v_{\max }$ from the width of the $21-\mathrm{cm}$ profile; $v_{\max }=\Delta \mathrm{v}_{21-\mathrm{cm}} / 2$ sin $i$. We have $21-\mathrm{cm}$ observations for 73 of the 96 galaxies $\left(\delta>-19^{\circ}\right)$ in the minimum bias subset. We observe $v_{\max }$ (antapex-apex) $=-15 \pm 27 \mathrm{~km} \mathrm{~s}^{-1}, i . e .$, no difference in the peak velocity of the rotation for apex and antapex galaxies (Fig. 5) Thus on the basis of diameters and peak rotational velocity we see no evidence that antapex galaxies are overluminous compared with apex galaxies.

To assure appropriate corrections to the magnitudes, correlations of HM with extinction in our Galaxy, with internal extinction in each galaxy, and of the variation of $M_{0}$ with Sc luminosity class have been calculated from the sample itself. The coefficient of galactic extinction, determined from the slope of the least squares fit of HM versus CsC $|b|$, is low (Fig. 6a); $A_{b}=0.15 \pm 0.03$, where $\Delta m_{b}=A_{b}(\csc |b|-1)$, and thus supports the low value 


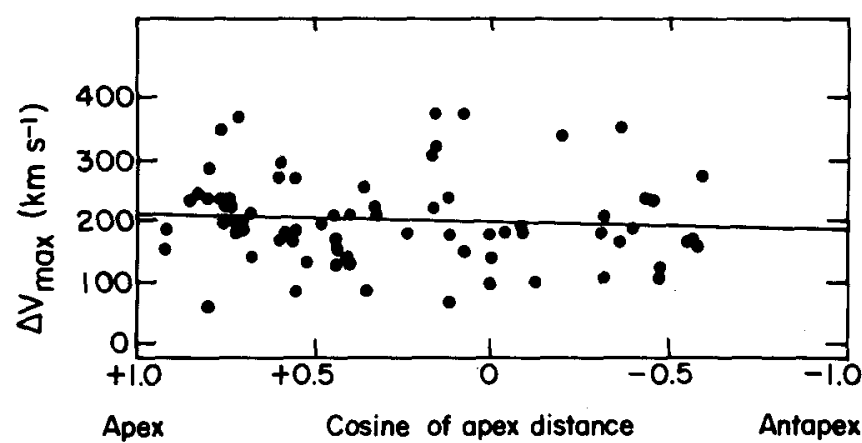

Fig. 5. Variation of $\mathrm{V}$ peak velocity of the rotation curve, as a function of cosine of apex distance. Least squares fit gives $\mathrm{V}_{\text {max }}=$ $199 \pm 10+(7.5 \pm 19) \cos \theta{ }^{\max }$ There is no evidence that antapex galaxies are more massive than apex galaxies.

generally derived from colors of stars. The effect of internal extinction in each galaxy is also low; $A_{i}=0.12 \pm 0.08 ; \Delta m_{i}=A_{i}(\sec i-1)$. This is in contrast
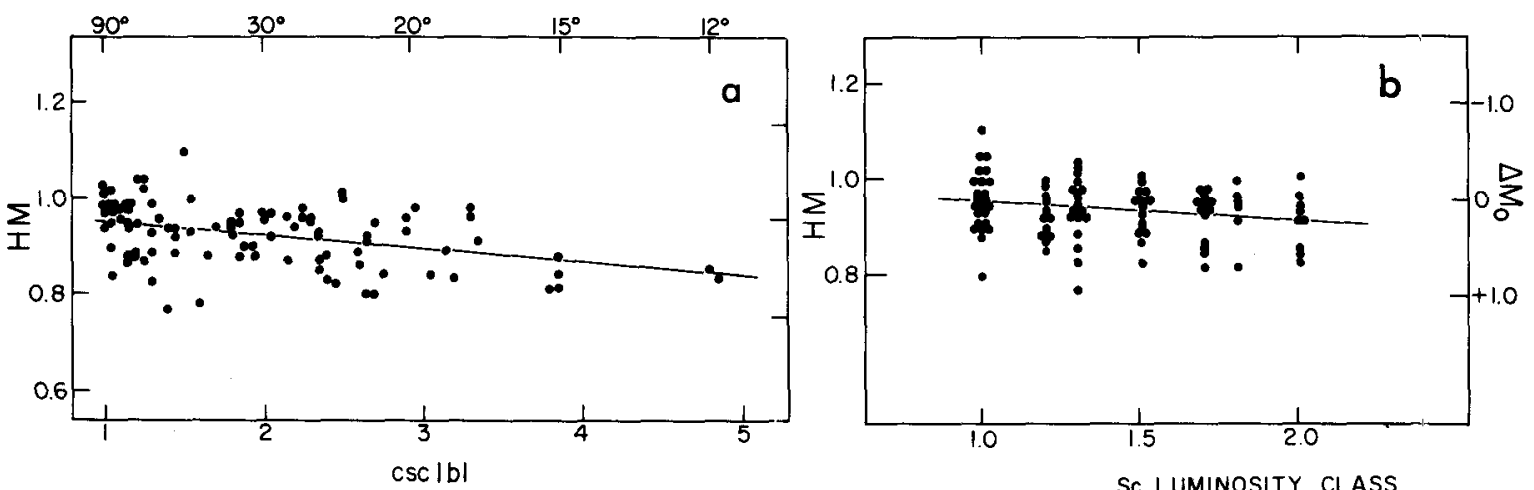

SC LUMINOSITY CLASS

Fig. 6. Correlation of HM with path length through the Galaxy. Line is linear least squares fit which gives $A_{0}=0.15 \pm 0.03$ (a). Correlation of HM with Sc luminosity classes SC I to Sc II. Note small change in $M_{0}$ over this range (b).

to the value 0.28 derived by Holmberg (1958) from galaxy surface brightnesses, and used in all current studies. Finally, the variation of $M_{0}$ with Sc luminosity class is very low (Fig. 6b): $A_{L C}=0.18 \pm 0.09 ; \Delta m_{L C}=A_{L C}(L C-1)$. This is smaller than the conventionally adopted value of 0.6 (van den Bergh 1960) or 1.0 (Sandage and Tammann 1974).

The 2l-cm magnitudes for the Sc sample, $m_{21}=[-2.5$ (integrated flux density)] have been used to investigate possible regional magnitude errors. Because the $21-\mathrm{cm}$ magnitudes are unaffected by galactic extinction, the variation of $m_{0}-m_{21}$ will map errors in the optical magnitudes. Examination of $m_{\circ}-m_{21}$ as a function of $\alpha$, of $\delta$, of galactic longitude, and of angular distance from the apex, gives no evidence for a grossly incorrect form for the galactic extinction, or for systematic errors in the magnitude system as a function of position on the sky greater than about 0.1 . The lack of 
significant variation of $\mathfrak{m}_{0}-m_{21}$ with galactic longitude and $\cos \theta$ are shown in Fig. 7

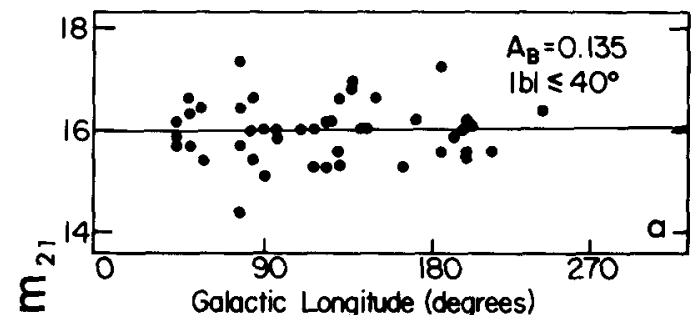

E

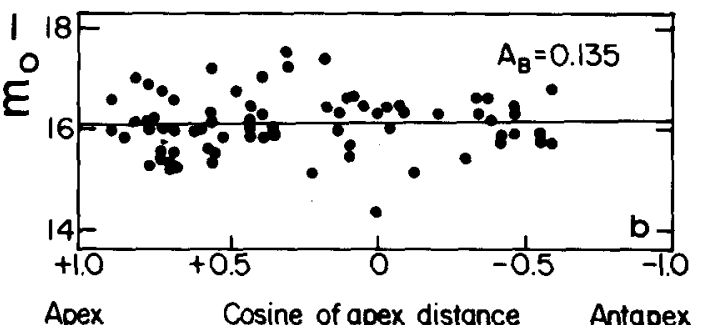

Fig. 7. Variation of $\mathrm{m}_{0}-\mathrm{m}_{21}$ as a function of galactic 21 longitude for galaxies $|\mathrm{b}|<40^{\circ}$. Least squares fit gives $m_{0}-m_{21}$ $=15.97 \pm 0.21-\left(5 . \times 10^{-5} \pm 1 . \times 10^{-3}\right) \quad \ell$, $0<\ell<180^{\circ}$.

(Lower) $m_{0}-m_{21}$ as a function of apex distance ${ }^{21}$ least squares fit gives $16.11 \pm 0.08$ $(0.03 \pm 0.15) \cos \theta$.

We believe that the Sc data have been handled with care to eliminate a Malmquist bias and systematic errors in the magnitudes, yet the anisotropy remains independent of the details of the analysis. Confirmation of this result (Rubin et al. 1975b) comes from Schechter (1976) who has used all 184 Sc galaxies in a simultaneous solution for 9 parameters, using an approach patterned after that of Gunn and oke (1975) to eliminate the bias. His results, equivalent to one in which the diameter-luminosity correction discussed above is not included (and consequently with higher errors) is:

$\mathrm{v}_{0}=715 \pm 250 \mathrm{~km} \mathrm{~s}^{-1}, \ell=184^{\circ}, \mathrm{b}=0^{\circ}$.

Sandage and Tammann $(1975 a, b)$ suggest that the Sc I anisotropy arises from a Malmquist bias due to density fluctuations. A variation on the model by Fall and Jones (1976) locates the galaxies in a near and far cloud; galaxies in the near cloud are then preferentially of low lunimosity. However, all explanations in terms of density fluctuations require that $M_{0}($ apex $) \neq$ $M_{0}$ (antapex). We believe that the procedures we have used to minimize the bias, coupled with the evidence that $M_{0}$ is similar for apex and antapex galaxies, makes this explanation unlikely. Doroshkevich and Shandarin (1976) attribute the anisotropy to velocity variations in dense "pancakes" (clusters) of galaxies, predicted from a non-linear graviational theory. The distance dependence of the variation of $\mathrm{HM}$ in the Sc sample makes this explanation unlikely.

(b) Other Studies

Analyses of other samples indicate values of $\langle H M\rangle$ which support the anisotropy observed in the Rubin et al. (1973) sample, according to their

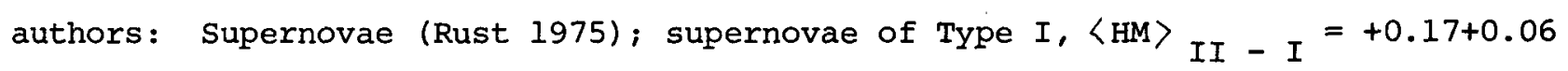


(Le Denmat and Vigier 1975); brightest cluster galaxies, supernovae, Seyfert galaxies (Jaakkola et al. 1976); compact galaxies from the Zwicky catalogue with absorption line velocities, 〈HM II _ I $=+0.17 \pm 0.09(13<\mathrm{m}<15)$, but decreasing to $\langle\mathrm{HM}\rangle_{\text {II }-\mathrm{I}}=+0.05 \pm .05$ at greater distances $(15<\mathrm{m}<18)$ (Jaakkola et al. 1975a). In all of these studies, conventional magnitude corrections have been applied (often the larger value for galactic extinction), generally little or no examination of the bias has been made, and the sky coverage ranges from poor to horrifying. For the supernovae studies, magnitude are generally available only after maximum light, adding an additional uncertainty. It is difficult to know how much weight to assign to these studies; these complex questions have not been examined. A study by Bahýl (1974) of the directional dependence of redshifts of galaxies deserves mention as an early, interesting attempt at a meaningful analysis.

The only additional sample of which I am aware which does have all-sky coverage is the sample of $E$ and so Galaxies of Sandage (1975), in the velocity range $3500<\mathrm{V}<6500 \mathrm{~km} \mathrm{~s}^{-1}$. Values of $\mathrm{HM}$ for these galaxies exhibit the same variation in amplitude and phase as do the Sc I galaxies. Sandage has argued that the lack of an enhanced effect for the nearer galaxies in his sample, $\mathrm{V}<3500 \mathrm{~km} \mathrm{~s}^{-1}$, makes this result questionable. However, there is a Malmquist bias in the E+SO sample, and the low velocity $(V<3500)$ galaxies have extremely poor sky distribution. Values of $\mathrm{H}$ decrease systematically with decreasing $\mathrm{V}:\langle\mathrm{H}\rangle=52.3 \pm 1.7$ for $3500-6500 \mathrm{~km} \mathrm{~s}^{-1}$, but $\langle H\rangle=47.6 \pm 2.3$ for $\mathrm{V}<2500 \mathrm{~km} \mathrm{~s}^{-1}$. Moreover, the sample contains only antapex galaxies, $\mathrm{V}<3500 \mathrm{~km} \mathrm{~s}^{-1}$. Hence the bias is very position dependent, so the low values of $\mathrm{H}$ in the antapex for the nearer galaxies tell nothing about an anisotropy, but only about a bias. I would conclude that the Sandage sample $3500<\mathrm{V}<6500$ $\mathrm{km} \mathrm{s} \mathrm{s}^{-1}$, does support the anisotropy found for Sc I galaxies.

Guthrie (1976) has analyzed the deviations from the Hubble magnitudes, $\delta \mathrm{m}$, for 42 brightest elliptical in clusters, covering slightly more than one half of the sky (Sandage and Hardy 1973). In the two regions defined by RFR, these galaxies exhibit a variation of $\delta m=0.4 \pm 0.1$. Because the sample is more distant than the Sc I sample, a variation of $\delta m=0.4$ (as observed in both samples) corresponds to a $\Delta V$ from apex to antapex of $2100 \pm 500 \mathrm{~km} \mathrm{~s}^{-1}$, or a motion of the Local Group of about $2100 / 2=1000 \mathrm{~km} \mathrm{~s}^{-1}$. The nearest and most distant galaxies in this sample too suffer a severe positional bias; eliminating these galaxies reduces the velocity to about $\mathrm{V} 800 \mathrm{~km} \mathrm{~s}^{-1}$. However, the distribution of $\mathrm{HM}$ across the sky (in the region observed) is not 
sinusoidal. Most high HM are $\sim 90^{\circ}$ from low HM values.

This result suggests that the anisotropy is not due to the motion of the observer, but arises from magnitude errors or a variation in $H$. As the $E$ galaxies have photoelectric magnitudes (Sandage 1972b, 1973; Peterson 1970), it is difficult to see how they could suffer the same errors as the Sc I sample, with magnitudes coming about equally from photoelectric observations (Graham 1976) and photographic magnitudes (Zwicky et al. 1961-1968).

Some fraction of the Guthrie sample is common to the Sandage (1975) E+SO sample, with more recent photometry. Because the more recent Sandage work does give evidence for an anisotropy, judgment on the Guthrie sample should be deferred until new photometry is available for the entire sample.

(c) The cluster sample of Weedman (1976)

In preparing this review, the most unexpected results came in examining the paper by weedman (1976). For nine rich clusters of galaxies covering only a small fraction of the sky, Weedman determined nuclear magnitudes (luminosites within radii of about $5 \mathrm{kpc}$ ) for the 10 brightest galaxies per cluster. After correction for aperture effects, k-diming, and galactic extinction, the nine points define a Hubble diagram which "shows no evidence for significant non-Hubble velocities for $1000<\mathrm{CZ}<11000 \mathrm{~km} \mathrm{~s}^{-1}$." This low scatter diagram is shown in Fig. $8 \mathrm{a}$.

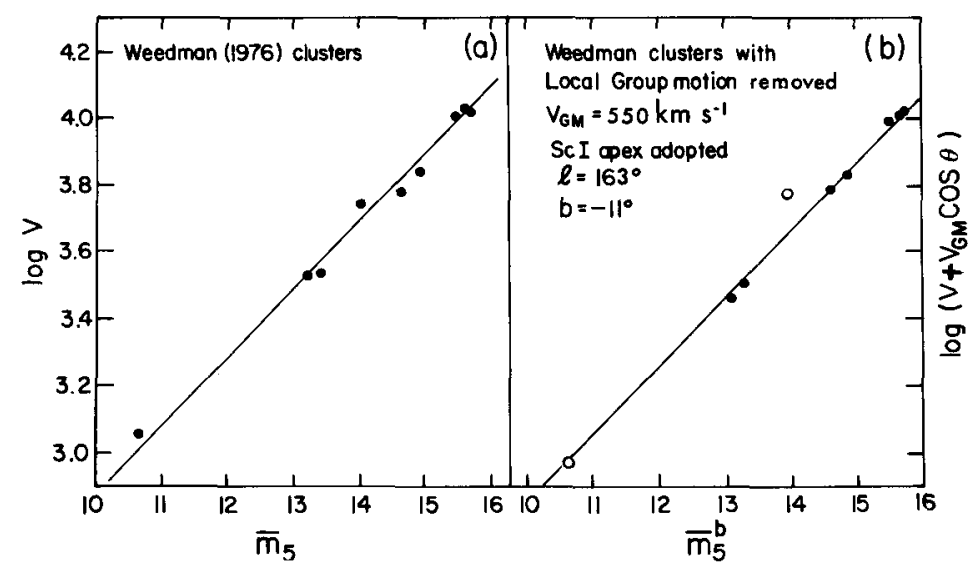

Fig. 8. (a): Hubble Diagram for rich clusters (Weedman 1976). (b): Same, with component of motion of the Local Group removed from each observed velocity; magnitudes are corrected for absolute magnitude bias. Hubble line is least squares fit with $\mathrm{HM}=0.868$.

However, with one outstanding exception, these clusters offer support for an asymmetry of HM of the same phase and amplitude as the Sc I galaxies (Rubin et al. 1976b). Within the small scatter, the residuals are not distributed at random on the sky. In Table II we list the values of $\mathrm{V}$ and $\overline{\mathrm{m}}_{5}$ (Weedman 1976), and the calculated values of $\mathrm{HM}=\log \mathrm{V}-0.2 \overline{\mathrm{m}}_{5}$. Note first 
TABLE II. Parameters for Weedman (1976) clusters

\begin{tabular}{lcccccc}
\hline Cluster & $\left(\mathrm{km}^{\mathrm{V}}{ }^{-1}\right)$ & $\overline{\mathrm{m}}_{5}$ & $\mathrm{HM}$ & $\overline{\mathrm{m}}_{5}{ }^{\mathrm{b}}$ & $\mathrm{HM}^{\mathrm{b}}$ & $\cos \theta+$ \\
\hline Virgo & 1120 & 10.64 & 0.921 & & & -0.312 \\
Centaurus & 3390 & 13.22 & 0.886 & 13.13 & 0.904 & -0.830 \\
Hydra I & 3450 & 13.40 & 0.858 & 13.31 & 0.876 & -0.332 \\
Perseus & 5470 & 13.97 & 0.944 & 13.95 & 0.948 & +0.973 \\
A1367 & 6120 & 14.58 & 0.871 & 14.58 & 0.874 & -0.089 \\
Coma & 6840 & 14.89 & 0.857 & 14.90 & 0.855 & -0.194 \\
Hercules & 10230 & 15.46 & 0.918 & 15.53 & 0.904 & -0.592 \\
Al185 & 10470 & 15.64 & 0.892 & 15.71 & 0.878 & +0.132 \\
A2147 & 10530 & 15.61 & 0.900 & 15.68 & 0.886 & -0.617 \\
& & & & & & \\
\hline
\end{tabular}

${ }^{\prime} \bar{m}_{5}^{b}=\bar{m}_{5}+0.32(\log \mathrm{V}-3.80) ; \mathrm{HM}^{\mathrm{b}}=\log \mathrm{V}-0.2 \overline{\mathrm{m}}_{5} \mathrm{~b}$.

$+\theta^{5}=$ angular distance from apex defined by Sc I galaxies.

that the Perseus cluster has an extremely discrepant $(>6 \sigma)$ value of $\mathrm{HM}$; for the other clusters, $\mathrm{HM}=0.888 \pm 0.009$. Note also that for the 4 clusters with $3390\left\langle\mathrm{~V}\left\langle 6940 \mathrm{~km} \mathrm{~s}^{-1},\langle\mathrm{HM}\rangle=0.868 \pm 0.007\right.\right.$, while for the 3 clusters near $\mathrm{V}=10000 \mathrm{~km} \mathrm{sec}{ }^{-1}\langle\mathrm{HM}\rangle$ is larger, $\langle\mathrm{HM}\rangle=0.904 \pm 0.008$. This is evidence of a Malmquist bias, even for the mean cluster magnitudes. The difference $\Delta \mathrm{HM}=0.04$ corresponds to a brighter intrinsic magnitude of 0.2 for the more distant galaxies. Even clusters suffer a Malmquist bias; a distant cluster will be preferentially observed if its brightest members are intrinsically brighter than those in a second equally distant cluster.

A plot of the values of HM for the galaxies with $\mathrm{V}$ near $5000 \mathrm{~km} \mathrm{~s}^{-1}$ and $\mathrm{V}$ near $10000 \mathrm{~km} \mathrm{~s}^{-1}$ is shown in Fig. 9a, as a function of angular distance

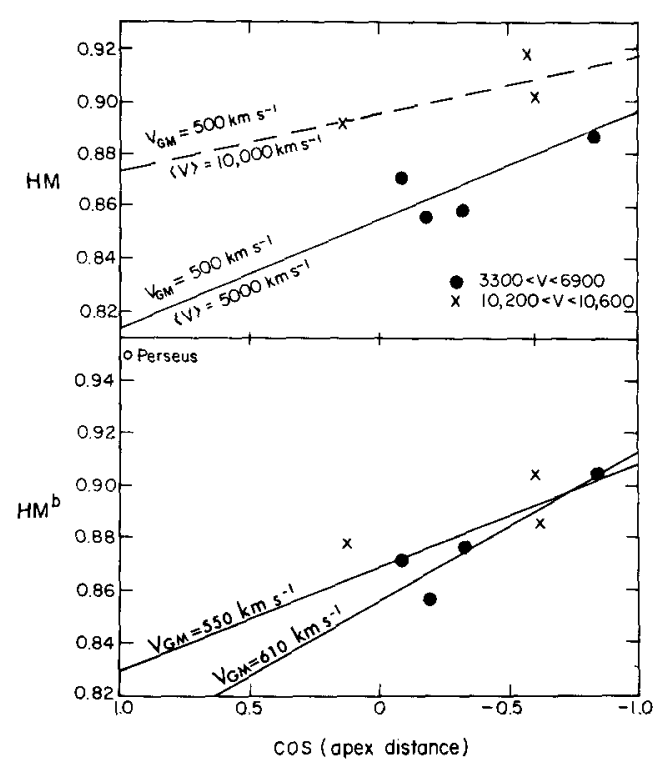

Fig. 9. (upper): Values of HM for 7 clusters, versus cos (apex distance); apex defined by Local Group motion with respect to Sc I galaxies. Solid line shows variation of $\mathrm{HM}$ for sample with $\mathrm{V}=5000 \mathrm{~km} / \mathrm{s}$, $\mathrm{V}_{\mathrm{GM}}=500 \mathrm{~km} / \mathrm{s}$. Dashed line shows variation of $H M$ for $\mathrm{V}=10000 \mathrm{~km} / \mathrm{s}$, $\mathrm{v}_{\mathrm{GM}}=500 \mathrm{~km} / \mathrm{s}$.

(lower): Values of $\mathrm{HM}$, corrected for bias, as a function of cos (apex distance). $\mathrm{V}_{\mathrm{GM}}=610 \mathrm{~km} / \mathrm{s}$ is least squares fit $\mathrm{to}_{4} 4$ points, $3300<\mathrm{V}<6900 \mathrm{~km} / \mathrm{s}$. $V_{\mathrm{GM}}=550 \mathrm{~km} / \mathrm{s}$ is fit to 7 points, $3300<\mathrm{V}<10600 \mathrm{~km} / \mathrm{s}$. 
from the apex determined for the SC I galaxies. Almost all the galaxies lie in the antapex hemisphere. For a Local Group velocity of $\mathrm{V}_{\mathrm{GM}}=500 \mathrm{~km} \mathrm{~s} \mathrm{~s}^{-1}$, the slope of the variation of $\mathrm{HM}$ with $\cos \theta$ for a sample near $\mathrm{V}=5000 \mathrm{~km} \mathrm{~s}^{-1}$ is shown by the solid line, and is a good fit to the few points. The same velocity, as it would be observed for a sample at $\mathrm{v}=10000 \mathrm{~km} \mathrm{~s}^{-1}$ is shown as the dotted line, through the high velocity points; it also does not violate the data. In order to be able to use all seven clusters in a single solution, we eliminate the bias in the sample as follows.

The increase in $\mathrm{HM}$ (i.e., $\mathrm{M}_{\mathrm{O}}$ ) with $\log \mathrm{V}$ is evaluated in a linear least squares fit:

$$
\mathrm{HM}=0.636 \pm 0.142+(0.065 \pm 0.037) \log \mathrm{V} \text {. }
$$

We then apply this variation as a correction to each $\bar{m}_{5}$ :

$$
\overline{\mathrm{m}}_{5}^{\mathrm{b}}=\overline{\mathrm{m}}_{5}+5(0.065)(\log \mathrm{v}-3.80) \text {, }
$$

where the constant in the parenthesis is chosen so that the correction will be zero at the mid-velocity. Values of $\overline{\mathrm{m}}_{5}{ }^{\mathrm{b}}$ and $\mathrm{HM}^{\mathrm{b}}$, both corrected for the bias, are listed in columns 5 and 6 of Table II, and plotted in Fig. 9b. It is apparent that these 7 clusters (Perseus and Virgo are discussed below) do not have values of HM which have a random distribution with respect to the apex determined for the Sc I galaxies, but show a significant correlation of HM with cosine apex distance. A linear least squares fit gives:

$$
\begin{aligned}
& \mathrm{HM}=0.856 \pm 0.009-(0.056 \pm 0.019) \cos \theta ; \mathrm{n}=4,3390<\mathrm{V}<6840 ; \\
& \mathrm{HM}=0.868 \pm 0.008-(0.038 \pm 0.016) \cos \theta ; \mathrm{n}=7,3390<\mathrm{V}<10530 . \\
& \text { This variation of } \mathrm{HM} \text { with } \cos \theta \text { corresponds to a Local Group motion: } \\
& \mathrm{V}_{\mathrm{GM}}=610 \pm 225 \mathrm{~km} \mathrm{~s}^{-1}, 3390<\mathrm{V}<6840 ; \\
& \mathrm{V}_{\mathrm{GM}}=550 \pm 250 \mathrm{~km} \mathrm{~s}^{-1}, 3390<\mathrm{V}<10530 .
\end{aligned}
$$$$
\mathrm{HM}=0.868 \pm 0.008-(0.038 \pm 0.016) \cos \theta ; \mathrm{n}=7,3390<\mathrm{V}<10530 \text {. }
$$

In both cases, the range in $\mathrm{V}$ has not been taken into account. More sophisticated analysis does not seem warranted in view of the few points. However, it seems valid to conclude that rich clusters do offer support for a motion of the Galaxy and the Local Group of the order of $\mathrm{V}_{\mathrm{GM}}{ }^{2500 \mathrm{~km} \mathrm{~s}} \mathrm{~s}^{-1}$.

When we remove from each observed velocity the component $v_{G M} \cos \theta$ due to the Local Group velocity, the resulting Hubble diagram is remarkably scatter-free, Fig. 8b. No bias correction is included for the Virgo cluster; there is no way to evaluate a bias for a single nearby cluster. Note however, that with $\cos \theta=-0.312$ for Virgo $\left(\theta=109^{\circ}\right)$, the contribution due to the component of the Local Group velocity is of order $+200 \mathrm{~km} \mathrm{~s}^{-1}$ in the observed Virgo velocities, if the cluster is not participating in the Local Group velocity. Removing this component decreases the cluster velocity 
and brings it close to the new Hubble line. The extreme deviation of the Perseus cluster, noted also by Weedman, is too large to be due to observational errors. Even a zero correction for extinction $\left(b=-13^{\circ}\right)$ would not place it on the line in Fig. 8b. The Perseus cluster is an extremely perplexing one.

This is a fine opportunity to point out the effect of a bias in a sample, as illustrated by Fig. 9a. Before correction for the bias, the scatter of the 7 values of HM with $\cos \theta$ camouflages any correlation. Had the 3 high velocity clusters been located near the apex (cos $\theta \sim 1$ ), a completely different relation would have been suggested. This shows graphically why a sample with a position dependent bias (or more properly, without allsky coverage in each velocity range) cannot be used to evaluate an isotropy or lack thereof in any sample.

In the Weedman statement concerning lack of evidence for "significant non-Hubble velocities", it is the word significant which tells the tale. For

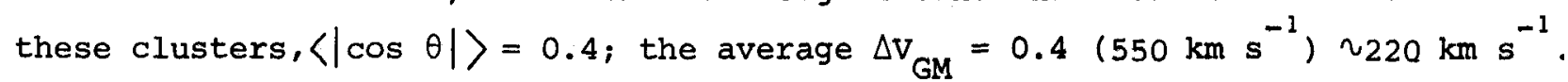
On the Hubble diagram, points move on the average only $\sim[\log (6300)-\log$ (6300-220)] or 0.02 units in $\log \mathrm{V}$, which corresponds to a distance equivalent to a diameter or two of a point. A Local Group velocity of $\sim 500 \mathrm{~km} \mathrm{~s}^{-1}$ produces only a very subtle effect on a Hubble diagram of galaxies with $3000<\mathrm{V}<10500 \mathrm{~km} \mathrm{~s}^{-1}$, if the clusters are not preferentially located toward the apex or antapex. Small scatter is not synonymous with isotropy.

In analyzing these data of Weedman, I have violated several conditions enumerated earlier: sky coverage is poor and the velocity range is large. Therefore, until better sky coverage in a smaller velocity range is available, these results must be interpreted with caution. But they point out the disastrous trap of attempting to use a limited sky sample to show that no anisotropy exists.

(d) The Diameter Study of Evans and Hart (1976)

Three parameters are generally available from observations of galaxies: velocities, magnitudes, and angular diameters. Taken two at a time, they can be used to define three moduli. Previously, we have used the velocitymagnitude modulus, which contains the least uncertainty in the observational quantities. The diameter velocity modulus, $\log v+\log d$, [i.e., $\log$ (linear diameter)], has been used by Evans and Hart (1976) to search for possible anisotropies in the sandage (1972a) elliptical galaxies, $1200<\mathrm{V}<$ $25500 \mathrm{~km} \mathrm{~s}^{-1}$. No statistically significant difference in the diameter modulus 
is observed, with the sky division of Rubin et al. (1973). However, the large range in $\mathrm{V}$, combined with the high velocity upper limit, makes it impossible to detect a motion of the observer as small as $\mathrm{v}_{\mathrm{GM}} \sim 500 \mathrm{~km} \mathrm{~s} \mathrm{~s}^{-1}$. Just as galaxies with $\mathrm{V}$ greater than 10000 carry little information concerning a Local Group motion of $500 \mathrm{~km} \mathrm{~s}^{-1}$, so also the differences in diameters for two galaxies with $\mathrm{V}=10000+500$ and $\mathrm{V}=10000-500 \mathrm{~km} \mathrm{~s}^{-1}$ is slight. In Table $I$, the difference $\Delta H M$ (column a) are identically equal to the differences predicted for the diameter modulus with increasing $V$. The lack of a detectable anisotropy over this large a velocity range reveals nothing about a Local Group velocity. It does imply that $\mathrm{H}$ does not vary across the sky over this distance.

If the sample can be divided into subsets by distance, if the diameterdistance bias is evaluated (far galaxies come preferentially from the "big. galaxy" tail of the diameter distribution), and if the decrease in galaxy diameter with galactic latitude is evaluated, then this procedure will offer a valuable addition to analyses of velocities and magnitudes.

\section{(e) Non-Doppler interpretations}

Numerous papers (references 6-II, Table III) have recently appeared, interpreting the observed anisotropies in terms of non-Doppler redshifts. Because several review papers at this colloquium will be devoted to nonDoppler effects, only very brief mention will be made here of this work.

One prevelant idea is reviewed by Jaakkola et al. (1976). In this model, light from distant galaxies is redshifted when passing through clusters of galaxies; this non-Doppler shift produces high values of HM in these regions. The authors show that the anisotropies observed for brightest cluster members, for supernova, and for Seyfert galaxies can be correlated with foreground clusters.

All of these samples have incomplete sky coverage, a very large range in velocity, and suffer serious bias problems. Moreover, only the correlation with "important" nearby clusters is included; for the very distant galaxies, $\mathrm{V} \sim 25000 \mathrm{~km} \mathrm{~s}^{-1}$, more distant clusters are not considered. Not until a proper observational sample is available, can this hypothesis be tested.

\section{PROBLEMS ARISING FROM ERRONEOUS MAGNITUDE CORRECTIONS}

Errors in all adopted corrections can produce regional systematic effects in the analysis. Le Denmat et al. (1975h) have pointed out that for the Sandage and Tammann $(1975 a, b)$ Sc galaxies, the calculated values for $\langle H\rangle$ 
increase from $\langle H\rangle=61.4 \pm 6.8$ for Sc $I$ to $\langle H\rangle=77.6 \pm 6.0$ for Sc III. This decrease in $\langle H\rangle$ with higher luminosity classes implies that $\Delta M_{0}=1.0$ is an overcorrection for the variation in $M_{0}$ from luminosity class I to II. With the van den Bergh (1960). value, $\Delta \mathrm{M}_{0}=0.6$, the decrease in $\mathrm{H}$ almost disappears, but a still smaller value seems implied. Additional studies of this effect have also been made: Jaakkola (1975), Le Denmat et al. (1975a), Jaakkola and Le Denmat (1976), and Bottinelii and Gouguenheim (1976).

Errors in the extinction correction could account for the observed anisotropy in HM for the SC I galaxies, if galaxies toward the anticenter region have 0.4 more extinction than those toward the center. Hartwick (1975) has examined the $B, V$, and $R$ colors of first ranked $E$ or so galaxies in clusters (Sandage and Hardy 1973) and concluded that there is a marginal effect, such that galaxies in the anticenter indicate an excess extinction of $0.15 \pm 0.11$ in $B ; 0.11 \pm 0.07$ in $\mathrm{V}$ and $0.08 \pm 0.08$ in $\mathrm{R}$. More observations, both optical and $21-\mathrm{cm}$, will be necessary to describe the longitude dependence of galactic extinction. The most recent study of the distribution of clusters of galaxies (Holmberg 1974) shows no evidence for increased extinction toward the anticenter.

In current isotropy studies which include spiral galaxies, no assessment has been made of the effect caused by the adoption of the Holmberg (1958) correction for internal extinction in each galaxy. If the low value determined for the Sc galaxies (Rubin et al. 1976a,b) is confirmed, it too will have repercussions for all such studies.

VII. THE ISOTROPY OF THE $3^{\circ} \mathrm{K}$ COSMIC BACKGROUND RADIATION

Experiments to determine the angular variation of the cosmic microwave background radiation have now been carried out at several wavelengths and with various techniques, but only over limited regions of the sky (Partridge 1974). Although the experimental techniques and interpretations are complex, they are consistent in showing a high degree of isotropy, implying an upper limit to the velocity of the sun, $\mathrm{v}_{\odot}<300 \mathrm{~km} \mathrm{~s}^{-1}$.

Results from the recent balloon flight of Corey and wilkinson (1976; Corey 1976) are shown in Fig. 10, along with the earlier measures of Henry (1970). A rotating radiometer measures the difference in radiation flux received by two horn antennas pointing $45^{\circ}$ from the zenith, $180^{\circ}$ apart in azimuth. From the difference in north minus south signal, and west minus east signal, Corey and wilkinson derive an upper limit for the motion of the sun $v_{\odot}<270 \pm 70 \mathrm{~km} \mathrm{~s}^{-1}, \alpha=13^{\mathrm{h}} \pm 2^{\mathrm{h}}, \delta=-25^{\circ} \pm 20^{\circ}$, with respect to the back- 


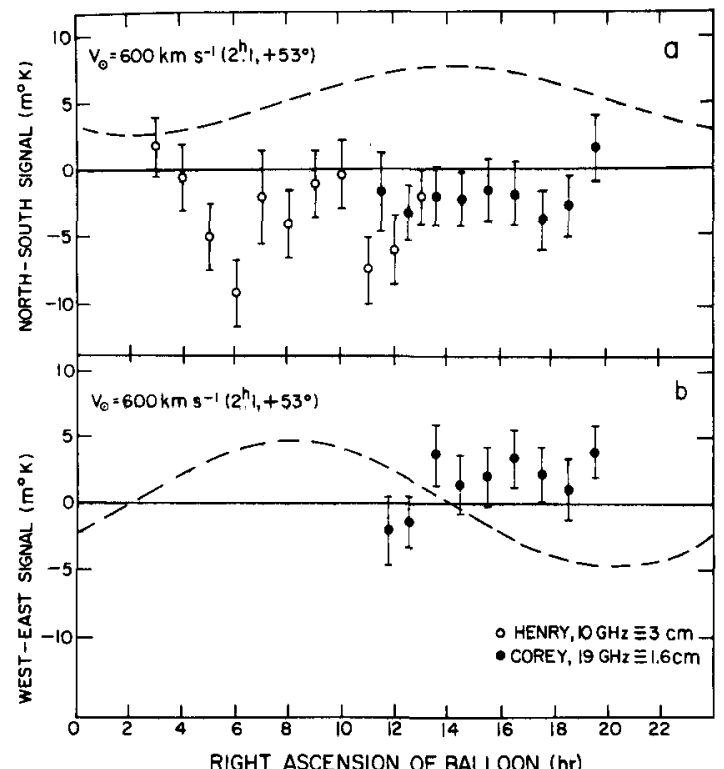

Fig. 10. (a): Difference in north minus south radiation flux as a function of right ascension, for Henry (1970) and Corey and Wilkinson (1976) balloon observations. Signal expected for $\mathrm{v}_{\odot}=600 \mathrm{~km} / \mathrm{s}, \alpha=2^{\mathrm{h}} 1, \delta=+53^{\circ}$ $\left(V_{G M} \sim 500 \mathrm{~km} / \mathrm{s}\right)$ is shown as dashed line. (b): Same, for west minus east radiation flux. I thank Corey and Wilkinson for making their results available prior to publication.

ground radiation. The predicted signal, for a motion of the sun $v_{\odot}=600 \mathrm{~km} \mathrm{~s}^{-1}$ toward $\alpha=2.1, \delta=+25^{\circ}$ (corresponding to a Local Group motion $\mathrm{v}_{\mathrm{GM}}{ }^{\mathrm{h}} \mathrm{500} \mathrm{km} \mathrm{s}^{-1}$ ), is shown as the dashed line. There is a clear conflict between the results from the optical observations and the microwave studies.

Measurements of the background radiation covering a significant fraction of the sky have not been made. If all-sky observations support the isotropy of the cosmic background, and if a motion of the Local Group $\mathrm{V}_{\mathrm{GM}}{ }^{200} \mathrm{~km} \mathrm{~s}^{-1}$ is confirmed, then the resolution of this conflict is bound to increase our knowledge of the universe. One can speculate that there might still be surprises in store when the all-sky microwave radiation is observed, or that the resultant of the solar velocity with respect to an increasingly distant sample of galaxies might be close to zero. The physical and philosophical implications of both these possibilities are great.

\section{CONCLUSIONS}

Before any extragalactic sample can be analyzed for a possible anisotropy, all position dependent corrections to velocities, diameters, and magnitudes must be applied. Among these corrections are conventional ones imposed by the physical characteristics of galaxies (internal and external extinction, luminosity and diameter dispersion, cluster richness), as well as those imposed by the selection process (Malmquist bias). Lacking this, the results will indicate merely the regional variations of these parameters, rather than a cosmological phenomenon. At the present state of observational cosmology, there are extremely few all-sky studies (or even almost all-sky) for which adequate examination of the necessary corrections has been made.

Table III summarizes a few of these studies. It indicates the type of 
Table III. Summary of anisotropy deduced from recent studies

\begin{tabular}{|c|c|c|c|c|c|c|c|c|}
\hline \multirow[t]{2}{*}{ Sample } & \multirow[t]{2}{*}{ Velocity } & \multirow{2}{*}{$\begin{array}{l}\text { Sky } \\
\text { Cover }\end{array}$} & \multirow{2}{*}{$\begin{array}{l}\text { Bias } \\
f(\theta)\end{array}$} & \multirow{2}{*}{$\begin{array}{l}\text { Aniso- } \\
\text { tropy }\end{array}$} & \multicolumn{3}{|c|}{ Interpretation } & \multirow{2}{*}{$\begin{array}{c}\text { Source } \\
\text { of } \\
\text { Data }\end{array}$} \\
\hline & & & & & $\begin{array}{c}\mathrm{v}_{\mathrm{GM}} \\
(\mathrm{km} / \mathrm{s})\end{array}$ & $\Delta \mathrm{m}_{0}$ & $\Delta \mathrm{H} / \mathrm{H}$ & \\
\hline Sc I & $3500-6500$ & good & absent & yes & 500 & 0.4 & 208 & 1 \\
\hline Sc I & $1600-3500$ & good & absent & yes & 500 & 0.8 & 40 & 1 \\
\hline cluster E+SO & $3500-6500$ & good & absent & yes & 500 & 0.4 & 20 & 2 \\
\hline cluster E+SO & $0-3500$ & poor & present & * & --- & $-\ldots$ & --- & 2 \\
\hline cluster $\mathrm{E}$ & $8000-16000$ & poor & absent & yes & $(800)$ & 0.4 & 20 & 3 \\
\hline rich clusters & $3000-11000$ & poor & present & yes & 550 & 0.4 & 20 & 4 \\
\hline diameters (E) & $1200-25000$ & good & present & no & not $\mathbf{s}$ & udied & 0 & 5 \\
\hline various & large & poor & present & yes & non-D & ppler & effects & $6-11$ \\
\hline $3^{\circ} \mathrm{K}$ background & $z \geq 10^{3}$ & poor & & no & $v_{0}<3$ & $0 \mathrm{~km} / \mathrm{s}$ & & 12 \\
\hline
\end{tabular}

\section{Source:}

1. Rubin et al. (1976) 5. Evans and Hart (1976) 9. Jaakkola et al. (1975b)

2. Sandage (1975) 6. Pecker et al. (1972) 10. Karoji and Nottale (1976)

3. Guthrie (1976) 7. Dautcourt (1975) 1l. Jaakkola et al. (1976)

4. Weedman (1976) 8. Karoji et al. (1975) 12. Corey and Wilkinson (1976)

\section{* indeterminate}

object, the degree of sky coverage, the presence or absence of a Malmquist bias, the presence or absence of an anisotropy, possible interpretations, and the initial source of the data. As is apparent, the interpretations are not mutually consistent. The Sc I galaxies, $3500<\mathrm{V}<6500 \mathrm{~km} \mathrm{~s}^{-1}$ (Rubin et al. 1976a,b), the brightest Elliptical and so galaxies in clusters (Sandage 1975), and rich clusters of galaxies (Weedman 1976) all exhibit an anisotropy which can be interpreted as (1) a Local Group motion, $\mathrm{V}_{\mathrm{GM}} \sim 500 \mathrm{~km} \mathrm{~s}^{-1}$ toward the same apex; or (2), a variation in $\mathrm{m}$ or $\mathrm{M}_{\mathrm{O}}$ of $0^{\mathrm{m}} 4$; or (3) an anisotropic expansion, with $\triangle \mathrm{H} / \mathrm{H} \sim 208$. Of these possibilities, all but a Local Group motion are ruled out by the nearer Sc galaxies. Moreover, for the Sc sample, an examination of the optical and $21-\mathrm{cm}$ magnitudes, of the linear diameters, and of the peak velocities of the rotation curves indicates no differences in the physical parameters for apex and antapex galaxies, at about the $0^{\mathrm{m}_{1}}$ level.

The interpretation as a Local Group motion, $\mathrm{V}_{\mathrm{GM}}{ }^{2500} \mathrm{~km} \mathrm{~s}^{-1}$, is inconsistent with the results of Guthrie, which from limited sky observations suggest a velocity difference of about $800 \mathrm{~km} \mathrm{~s}^{-1}$ in two regions separated by about $90^{\circ}$, or a magnitude difference of 0.4 for these same regions. Even more severe, however, is the conflict with the isotropy of the $3^{\circ} \mathrm{K}$ cosmic background radiation, which requires $\mathrm{v}_{\odot}<300 \mathrm{~km} \mathrm{~s}^{-1}$. These problems notwithstanding, substantial evidence for a Local Group motion is now accumulating. 
The original purpose of the Sc galaxy program was to examine the isotropy of the Hubble expansion. The anisotropy which was discovered has now been interpreted as a motion of our Galaxy and the Local Group. When we remove this component of motion from the observed velocities, the Hubble flow is very uniform. A uniform Hubble flow, at about $10 \%$ level, has been found for other samples by Sandage and Tammann (1975a). For the Sc galaxies, after correction for magnitude effects and the Local Group velocity, the dispersion on the Hubble diagram is very small; $\sigma(\mathrm{HM})=0.055$ (Rubin et al. 1976a, b), or $\Delta H / H \sim 58$. No anisotropy above this level is observed in the Hubble expansion as deduced from the high luminosity spiral galaxies.

Permission to use Figures previously published in Astronomical Journal is greatfully acknowledged.

\section{References}

Bahýl, v. 1974, Bull. Astr. Inst. Czech., 25, 115.

Bergh, van den, S. 1960, Ap. J., 131, 215.

Bottinelli, L. and Gouguenheim, L. 1976, in preparation.

Corey, B. E. 1976, Ph.D. Thesis, Princeton University.

Corey, B. E. and Wilkinson, D. T. 1976, Bull. AAS., 8 , 351.

Dautcourt, G. 1975, Astr. and Ap., 38, 335.

Doroshkevich, A. G. and Shandarin, S. F. 1976, M.N.R.A.S., 175, 15p.

Evans, A. and Hart, D. 1976, Nature, 259, 469.

Fall, S. M. and Jones, B.J.T. 1976, preprint.

Graham, J. A. 1976, A. J., 81, (Sept.).

Gunn, J. E. and Oke, J. B. 1975, Ap. J., 195, 255.

Guthrie, B.N.G. 1976, Astrophy. and Sp. Sci., in press.

Hartwick, F.D.F. 1975, Ap. J. (Letters), 195, L7.

Henry, P. S. 1970, Ph.D. Thesis, Princeton University.

Holmberg, E. 1958, Medd. Lunds. Obs. Ser. 2, No. 136.

Holmberg, E. 1974, Astr. and Ap., 35, 121.

Hubble, E. 1929, Proc. Nat Ac. Sci. (U.S.), 15, 168,

Jaakkola, T. 1975, C.R.A.S. Paris, 281B, 49.

Jaakkola, T. Karoji, H., Moles, M., and Vigier, J. P. 1975, Nature, 256, 24.

Jaakkola, T., Moles, M., Vigier, J. P., Pecker, J. C. and Yourgrau, W. 1975b, Found. of Phys, $\underline{5}$, No. 2 .

Jaakkola, T., Karoji, H., LeDenmat, G., Moles, M., Nottale, L., Vigier, J. P., and Pecker, J. C. 1976, M.N.R.A.S., in press.

Jaakkola, T. and LeDenmat, G. 1976, in press. 
Karoji, H., Nottale, L., and Vigier, J. P. 1975, C.R.A.S. Paris, 287, 409. Karoji, H., and Nottale, L. 1976, Nature, 259, 31.

Le Denmat, G. and Vigier, J. P. 1975, C.R.A.S. Paris, 280B, 459.

Le Denmat, G., Moles, M., and Nieto, J. L. 1975a, Astr. and Ap., 45, 423.

Le Denmat, G., Moles, M., Nieto, J. L., and Vigier, J. P. 1975b, Nature, 257 , 773.

Partridge, R. B. 1974, in Confrontation of Cosmological Theories with

Observational Data, ed. M. S. Longair (Reidel: Boston), p. 157.

Pecker, J. C., Roberts, A. P., and Vigier, J. P. 1972, Nature, 237, 227.

Peterson, B. A. 1970, A. J., 75, 695.

Rubin, V. C., Ford, w. K. Jr., and Rubin, J. S. 1973, Ap. J. (Letters), 183, L111.

Rubin, v. C., Ford, w. K. Jr., Thonnard, N., Robert, M. S., and Graham, J. A. 1976a, A. J., 81, (Sept.), (Paper I).

Rubin, V. C., Thonnard, N., Ford, W. K. Jr., and Roberts, M. S. 1976b, A. J., 81, (Sept.), (Paper II).

Rust, B. W. 1975, Bull. A.A.S., No. ㄱ, 236.

Segal, I. 1975, Proc. Nat. Ac. Sci. (U.S.), 72, 2473.

Sandage, A. R. 1972a, Ap. J., 173, 485.

Sandage, A. R. 1972b, Ap. J., 178, 1.

Sandage, A. R. 1973, Ap. J., 183, 711.

Sandage, A. R. 1975, Ap. J., 202, 563.

Sandage, A. R. and Hardy, E. 1973, Ap. J., 183, 743.

Sandage, A. R. and Tammann, G. 1974, Ap. J., 194, 559.

Sandage, A. R, and Tammann, G. 1975a, Ap. J., 196, 313.

Sandage, A. R. and Tammann, G. 1975b, Ap. J., 197, 265.

Schechter, P. 1976, private communication.

Teerikorpi, P. 1975a, Obs., 95, 105.

Teerikorpi, P. 1975b, Astr. and Ap., 45, 119.

Vaucouleurs, G. de 1972, in External Galaxies and Quasi-Stellar Objects, ed.

D. S. Evans (Springer-Verlag: New York), p. 353.

Weedman, D. W. 1976, Ap. J., 203, 6.

Zwicky, F., Herzog, E. Wild, P., Karpowicz, M., and Kowal, C. T., 1961-1968.

Catalogue of Galaxies and Clusters of Galaxies, in 6 Vols. (Pasadena: California Institute of Technology). 


\section{DISCUSSION}

I.E. SEGAL: There is an additional explanation for the Rubin-Ford anomaly, to which reference has not yet been made. This is that the chronometric cosmology is valid. In this model the expected magnitude difference between intrinsically similar objects at different low redshifts is half of that anticipated on the Hubble law. The observed notably slight apparent magnitude difference between the two galaxy groups differs from this value by $\sim 2 \sigma$, which is not significant, particularly in view of the ex post facto nature of the specification of the regions of the sky involved; although it appears to differ significantly from the Hubble law prediction. The situation is similar, but more complex, as regards the apparent motion of the sun.

V. RUBIN: Our data will shortly appear in print (Sept. 1976, Astron. J.), and I welcome an analysis by Dr. Segal and others in terms of various models.

J.C. PECKER: The bias mentioned by Tammann (and Sandage) and by Rubin is assumed to be due mostly to dispersion in actual absolute magnitudes. But a possible source of dispersion which pushes the effect the other way around could be the existence of "non-Doppler" apparent velocities, which we do not want to exclude a prioi at least! Moreover, it has been shown that the Karoji-Nottale (KN) effect was not due to the Malmquist bias (Nature, 259, 31).

The anisotropy found by Karoji, Nottale, and others, cannot be represented by any motion, as it has a morphology of the "porcupine" type, quite different of the two-directional anisotropy found by Rubin and Ford. We ( $K, \mathbb{N}$ et. al. Mon. Not. Roy. Astr. Soc., in press) think the RubinFord effect is resulting from averages on KN effects concerning objects at the same distance. The $\mathrm{KN}$ anisotropy still exists at larger distances as the Rubin-Ford anisotropy disappears.

V. RUBIN: It seems most likely that the anisotropy discussed by KN is not the same as that observed by us.

J.M. BARNOTHY: We have investigated in the two Rubin-Ford regions the Hubble plot of 141 quasars which have photoelectrically determined U,B,V magnitudes, and found $\mathrm{HM}_{\mathrm{II}}-\mathrm{HM}_{\mathrm{I}}=-0.17 \pm 0.07$. However the redshift in 
region $I$ is the larger.

V. RUBIN: If the anisotropy which we observe arises because of the motion of the observer, then it would not be observable in quasars with large $z$, because of the reasons discussed in the talk. Hence it is not surprising that quasars with large $z$ and a sample with a large range in $z$ does not support the anisotropy of the relatively nearby galaxies. 